\title{
Non-Communicable Diseases in Developing Countries: Causes and Health Policy/Program Assessments
}

\section{Tetsuji Yamada ${ }^{1 *}$, Chia-Ching Chen ${ }^{2}$, I-Ming Chiu ${ }^{3}$ and Syed W Rizvi ${ }^{3}$}

${ }^{1}$ Department of Economics, Center for Children and Childhood Studies, Rutgers University, New Jersey, USA

${ }^{2}$ Department of Epidemiology \& Community Health, School of Health Sciences \& Practice, New York Medical College, USA

${ }^{3}$ Department of Economics, Rutgers University, New Jersey, USA

\begin{abstract}
Objectives: This study focuses on non-communicable diseases (NCDs) and evaluates the effectiveness and efficiency of government public health policies on cardiovascular diseases and diabetes deaths per 100,000 population with age-standardized estimate of adults aged 25 and above of 30 South Asian and 46 African countries. Findings suggest that government policy implementation with focus on the reduction of deaths from NCDs is more efficient than general public health policy.
\end{abstract}

Methods: The study employed theoretically well founded PRECEDE-PROCEED model to assess effectiveness of public health programs. Three causal factors of NCDs and three policy issues constituted the core of this model. The causal factors included: body mass index (BMI) 30 \& over; systolic blood pressure; and total cholesterol. The policy issues included: health-related economics; health-related infrastructure; enabling, reinforcing and predisposing factors. Multiple regressions with robust method were used to assess effectiveness and concentration index for efficiency. The data for this study was taken from World Heath Statistics: 2008-2010.

Results: One percentage increase in BMI 30\&more raises 3.829 deaths from NCDs per 100,000 populations by country and the increase in NCDs depend on the size of population. One dollar per capita per year increase in government healthcare expenditures reduces NCDs by about 791 persons per 100,000 per year. An increase of $10 \%$ in government healthcare expenditures leads to $0.54 \%$ reduction in deaths from NCDs. The cost of this reduction in deaths from NCDs is $\$ 12.15$ per capita per year.

Implementation of key activities related to NCDs management reduces deaths from NCDs by 0.073 persons per 100,000 populations. An increase of $10 \%$ in NCD management expenditures leads to a $0.21 \%$ reduction in deaths or a decrease of 8.921 million deaths from NCDs. It costs $\$ 25.72$ per person per year. The estimated effect of cardiovascular diseases and diabetes management plan is 113.828 less NCD deaths per 100,000 populations than in countries without a plan to fight against NCDs. For efficiency, the concentration indices reveal that the NCDs management is more cost efficient than the government general healthcare expenditures.

Conclusions: In general, government policies and programs are found effective against NCDs. However, policies and programs focused on the reduction of NCDs are more efficient than general public health initiatives. A wellconstructed tax system for financing policy/program to deal with NCDs and related deaths is required.

\section{Introduction}

One of the major challenges that we are faced with in the international public health arena is the recent increase in non-communicable diseases (NCDs) in developing as well as industrialized countries. This scourge is in addition to already existing disparities and unequal distribution of healthcare services across developing countries. However, chronic diseases account for about $60 \%$ of the 565 million total deaths in the world. The share of the burden of NCDs in these deaths is about $46 \%$ of the global burden of these diseases in developing countries [1-5]. This proportion of NCD-related deaths is expected to increase to $57 \%$ from $46 \%$ by 2025 , and far too many will suffer from preventable diseases and health conditions [4-7]. Today, there is a 36 years gap in life expectancy between developing and industrialized countries. For example, a child born in Malawians expects to live for 47 years, whereas a child born in Japan may live as long as 83 years. Also, there is a large disparity between developing and industrialized countries regarding numbers of younger-age deaths from NCDs [8]. This is partly due to inadequate attention to their physical or environmental surroundings and government strategies, and partly due to their circumstances in which they are born, live, work, and get age $[1,5,9]$.

The disparity in health and life expectancy between developing and industrialized countries, especially in relation to NCDs, is due to the absence of well-organized healthcare delivery systems [1]. There is a need to increase multi-sector partnerships among communities, respective governments, and domestic and international agencies working on health projects. Studies indicate that in such partnerships stakeholders share their best practices to increase collaborative efforts to execute cost-effective and socially acceptable healthcare services in the needy neighborhoods and communities $[7,10]$. The expectation from such partnerships is the elimination of health disparities and application of effective strategic interventions aimed at improving quality of life of underserved population on priority basis. This suggestion is based on the findings that weak organizational structures of isolated organizations operational under governments in developing

*Corresponding author: Tetsuji Yamada, Department of Economics, Center for Children and Childhood Studies, Rutgers University, New Jersey, USA, Tel: 1-856225-6025; E-mail: tyamada@crab.rutgers.edu

Received July 22, 2013; Accepted September 02, 2013; Published September 04, 2013

Citation: Yamada T, Chen CC, Chiu IM, Rizvi SW (2013) Non-Communicable Diseases in Developing Countries: Causes and Health Policy/Program Assessments. J Trop Dis 1: 117. doi:10.4172/2329-891X.1000117

Copyright: @ 2013 Yamada T, et al. This is an open-access article distributed under the terms of the Creative Commons Attribution License, which permits unrestricted use, distribution, and reproduction in any medium, provided the original author and source are credited. 
countries do not show evidences of policy impacts on NCDs $[1,11]$. Hence, there is need of this intervention.

To achieve these objectives of health improvement plans, mainly in developing countries, the cooperating agencies need to identify and incorporate social determinants into their broader healthcare delivery and health promotion programs to reach the socially disadvantaged and underserved communities. The assumption is that if governments handle NCDs well, under structuralized cross-national communities in the public health framework, more progress may be expected. However, results obtained from previous research or freshly dug out from existing official policy documents, need to be reexamined for better interpretation and understanding of causes of failures as program managers have not yet been able to check NCDs at its onset. Though individuals aware of their lifestyle proximity to obesity, diabetes, and cardiovascular diseases turn to curative and preventive initiatives to escape NCDs, but inconsistencies in the process of program implementation and intermittent shifts in priorities have been unkind to them and to program objectives. Therefore, commitment of governments and other stakeholders in the struggle against NCDs to make concerted efforts to maintain continuity of initiatives is indispensable to achieve positive and sustained results.

There are fundamental issues common for developing countries to deal with. Many non-communicable diseases can be prevented by simply reducing smoking, cutting down alcohol intake, increasing physical inactivity, and avoiding unhealthy diet. This overall change in lifestyles is also likely to reduce high cholesterol, high blood pressure, diabetes, heart diseases, stroke, and lung cancer. Breast, colorectal, and stomach cancers are also preventable through periodical health checkups. The research literature on health issues shows that disparity and inequality in healthcare services and the resultant inaccessibility to healthcare facilities is closely related to income disparity and socioeconomic hardships of underserved population. In conditions of economic disparity and limited formal and informal health education, focused healthcare interventions by local governments are vital in a community-setting framework to mitigate disparity in healthcare services, and to generate good health outcomes in developing countries. Along with these measures, understanding of disparity outcomes is crucial if evaluation of health policies and programs is intended. To achieve these objectives, studies are needed to assess the governments' policy/program effectiveness on controlling non-communicable diseases and associated health issues.

This study explores the effects of public health policies/programs on NCDs. However, previous studies underscore that government policies are ineffective [1] and await improvement. Previous studies, to a great extent, lack credible and dependable assessment of government policies and present challenges to developing countries, with at-risk population in general, to examine what went wrong and where.

\section{Materials and Methods}

Majority of the total chronic disease deaths are attributed to cardiovascular diseases, diabetes, heart disease, and obesity [5]. The prevalence trend of these diseases is worrying. It is not only because these diseases have already begun to affect a large proportion of the population in developing countries, but because they have begun to surface at the early age of an individuals' lifestyle. This study focuses on NCDs (cardiovascular diseases and diabetes) and public health policies of developing countries.

The line of this research on NCDs and its policy implications

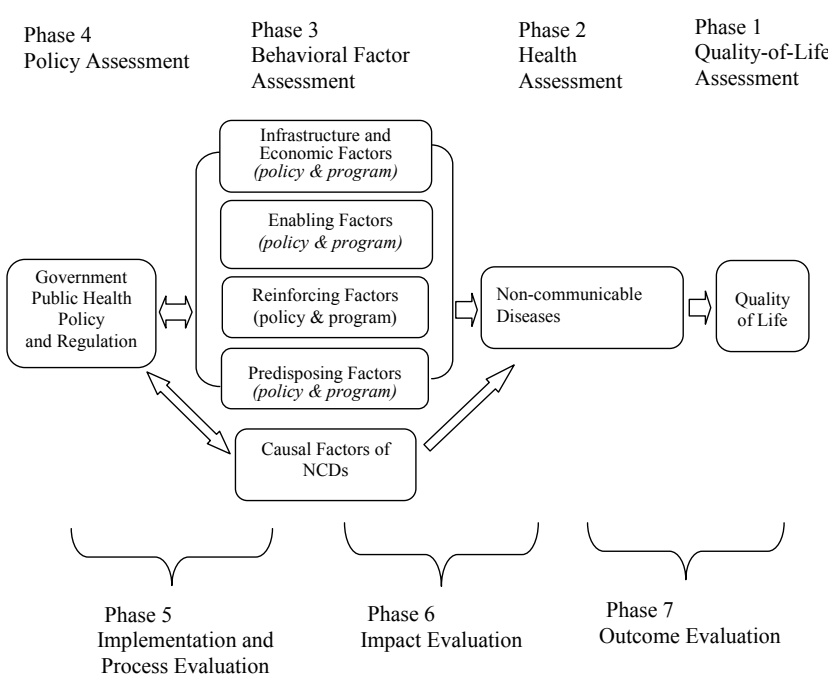

Notes: The Precede-Proceed model: Green \& Kreuter [12] is a planning model used to analyze and assess intervention/promotion for behavioral/achievement changes within a given socioeconomic-demographic environment. This model allows for a series of assessments and evaluations designed to help the policymakers to move from the recognition of problems and needs to decrease NCDs through the analysis of needs and problems.

Figure 1: Application of the PRECEDE-PROCEED Model to non-communicable diseases and preventive policies.

originates from the PRECEDE-PROCEED model (PP model) [12,13] This model offers some concepts and analytical tools to analyze public health policy choices. It also facilitates evaluation of government policies/programs between Phase 4 and Phase 6 of the PP model and brings to our understanding the "effect of government public health policy program" shown at the bottom of Figure 1. Phases 1-7 show the assessment of public health policy choices by the PP model. The focus of this study, at this stage, is on the assessment of five categories of influencing factors: economic and infrastructure, enabling, predisposing, and reinforcing factors by controlling causal factors of NCDs in Phase 3. The enabling factor (EN) is related to accessibility to preventing and curing NCDs under the healthcare system. The reinforcing factor (RE) is associated with management and operational policy/strategy/actions by the government.

The predisposing factor (PR) is connected to knowledge and information which encourage people to change risky health behavior. The key exposure variables are the causal factors of NCDs which are explained as cardiovascular and diabetes deaths per 100,000 population with age-standardized estimate among adults aged 25 and above by country -- 30 South Asian and 46 African countries. The measure of three causal factors of NCDs includes (1) percentage of identified population with a BMI 30 and higher with aged-standardized estimate among adults aged 25 and above by country; (2) systolic blood pressure trend age-standardized estimate among adults aged 25 and above by country; and (3) total cholesterol trend with age standardized among adults aged 25 and above by country. The explanation of policy programs includes government healthcare expenditures, health related infrastructure, and directly related government health policy and program for NCDs in Table 1.

\section{Data and measures}

The data for this study is taken from World Heath Statistics [5]. The main data are from the year 2008 with some additional data from 2009 


\section{Dependent variable}

Non-communicable disease: Cardiovascular and diabetes deaths per 100,000 population (age-standardized estimate among adults) by country (10,0000): mini=531.00; $\max =1650.00 ;$ mean=849.58.

\section{Independent variables}

\section{Causal factors of NCDs}

BMI 30 \& higher (\%): population with a body mass index (BMI) 30 and higher (age-standardized estimate among adults aged 25+ years) by country: mini=1.15; $\max =71.10 ;$ mean=14.32.

Systolic blood pressure: Systolic blood pressure trends (age-standardized estimate among adults aged $25+$ years) by country: mini=120.70; max $=137.45 ;$ mean=130.22. Total cholesterol: Total cholesterol trends (age-standardized among adults aged $25+$ years) by country: $\operatorname{mini}=3.90 ;$ max $=5.30 ;$ mean=4.44

\section{Health related infrastructure and economic factors (policy \& program)}

Improved sanitation (\%): Environmental sustainability/risk factors: proportion of the total population using improved drinking water sources and improved sanitation facilities $(\%)$ by country: $\operatorname{mini}=29.00 ; \max =100.00 ;$ mean $=61.78$.

Poverty (\%): Poverty lines: the overall percentage of the population living on under $\$ 1$ (Purchasing Power Parity current $\$ U S)$ a day by country: mini=5.88; max=81.29; mean $=42.49$.

Government healthcare expenditures (\$US): Reciprocal per capita general government spending on healthcare services at average exchange rate (Purchasing Power Parity current $\$$ US) per person per year by country: $\operatorname{mini}=0.058 \mathrm{e}-3$; $\max =0.277$; mean=0.058 [=121.448 with no reciprocal].

Enabling factors (policy \& program)

General availability of chemotherapy in the public health system: Health systems resources/capability: general availability of chemotherapy in the public health system by country (yes/no): $\operatorname{mini}=1 ; \max =2 ;$ mean=1.32.

Ministry of Health with responsibility for NCDs: Health systems resources/capability: Whether or not the country has a unit, branch or department in the Ministry of Health (or equivalent) with responsibilities for NCDs. (yes/no): $\operatorname{mini}=1$; $\max =2 ;$ mean=1.76.

General availability of diabetes and electrocardiogram at primary healthcare level: Health systems resources/medical testing: whether or not the country has diabetes testing (by blood glucose measurement, OGTT, or $\mathrm{HbA1c}$ ) generally available and electrocardiogram (ECG or EKG) generally available at the primary health care level. (2 4 which is higher): $\operatorname{mini}=2 ; \max =4$; mean=3.12.

Reinforcing factors (policy \& program)

Implementing NCD management (\$US): Implementing key activities related to NCD managements with per capita health expenditures (purchasing power parity current \$US): $\operatorname{mini}=22.33 ; \max =3042.53$; mean=257.20.

Cardiovascular \& diabetes diseases operational policy/strategy/action: NCD management: whether or not the country has an operational policy, strategy or action plan for cardiovascular and diabetes diseases. (yes/no): $\operatorname{mini}=1 ; \max =2 ;$ mean=1.29.

\section{Predisposing factor (policy \& program)}

Fiscal interventions for knowledge to influence behavior change: NCD management: the country is implementing any fiscal interventions for knowledge to influence behavior change and guidelines, protocols or standards available for the management of NCDs. (2 5 which is higher): mini=2; max=5; mean=3.12.

Table 1: Definition and descriptive statistics of variables (mean, minimum and maximum) used in the NCDs of South Asia and Africa analysis (observations=76).

and 2010. Some missing observation data is imputed by using the Stata imputation method with the exogenous variables in the framework of this study. The observations comprise 30 developing countries in South Asia and 46 developing countries in Africa. The total of observations is 76 including a dummy for the two groups of developing countries. The study does not separate two groups of developing countries because the nature of observation is small in size.

There are four key variables that are important measures for this analysis: health related infrastructure and economic factors (policy \& program); enabling factors (policy \& program); reinforcing factors (policy \& program); and predisposing factor (policy \& program) in Table 1. The study evaluates government policy impacts on cardiovascular and diabetes deaths (NCDs) per 100,000 population in 2008 with age-standardized estimate of male and female adults of developing countries in South Asia and Africa. Three causal factors are that (1) health status is the percentage of identified population with a body mass index (BMI) 30 and higher with age-standardized estimate of male and female adults aged 25 and above; (2) blood pressure is mean systolic blood pressure trends with age-standardized estimate among female and male adults aged 25 and above (3) cholesterol is mean total cholesterol trends with age-standardized among female and male adults aged 25 years and above by country.

\section{Statistical analysis}

The study assumes that the health outcome (NCDs) in Phase 6 (Figure 1) is attributed to the result of government public health policies. This is influenced by infrastructure and economic factors and by predisposing, reinforcing, and enabling factors in Phase 3. A functional structure in Phases 3 and 6 could be expressed as follows:

$\mathrm{NCDs}_{\mathrm{i}}=f\left(\mathrm{CF}, \mathrm{HR}, \mathrm{EN}_{\mathrm{i}}, \mathrm{PR}_{\mathrm{i}}, \mathrm{RE}_{\mathrm{i}}\right)+\mathrm{e}_{\mathrm{i}, \mathrm{NCD}}$
Equation (1) represents the relationship between the decrease in NCDs of country "i," and government public health policies in Phases 3 and 6 as seen in Figure 1. $e_{\mathrm{NCD}, \mathrm{i}}$ is an unobserved error, generally assumed to satisfy $\mathrm{E}\left(\mathrm{e}_{\mathrm{NCD}}, \mid \mathrm{CF}, \mathrm{HR}, \mathrm{EN}, \mathrm{PR}, \mathrm{RE}\right)=0 . \mathrm{CF}$ and $\mathrm{HR}$ represent causal factor of NCDs and health related economic/infrastructure factors respectively as shown in Table $1 . \mathrm{e}_{\mathrm{NCD}}$ is an unobserved error, generally assumed to satisfy $\mathrm{E}\left(\mathrm{e}_{\mathrm{NCD}} \mid \mathrm{CF}, \mathrm{HR}, \mathrm{EN}, \mathrm{PR}, \mathrm{RE}\right)=0$. Similarly, the outcomes of NCDs are incorporated in the PP model framework to examine the policy effects on NCDs in Figure 1. Table 1 explains variables. The baseline of the statistical analysis is a multiple regression with robust Stata program. The study examines policy effectiveness to find a threshold level of NCDs at the current given level of economic development and the level by including a reciprocal policy variable, i.e. government healthcare service expenditures [14]. In addition, the policy cost disparity is evaluated by using the concentration index to evaluate policy efficiency [15].

\section{Results}

\section{Reliability}

Table 1 presents explanation and descriptive statistics of the study sample. The variable of government healthcare expenditures shows small mean numbers in health related infrastructure and economic factors (policy \& program). It represents a reciprocal regressor of policy variable to evaluate threshold of NCDs by government policies for the regression model (Equation 1). For a poverty variable, the large percentage of population of some country, $81.29 \%$, lives under $\$ 1$ per day (PPP current \$US) and the mean of this value is $42.49 \%$. Thus, the population of many countries is extremely indigent according to the U.S. standard. For causal factors of NCDs, the mean of BMI 30 \& higher is $14.32 \%$ which is not extremely high. However, some countries have $71.1 \%$ obese population aged 25 and above. The study finds that 


\begin{tabular}{|c|c|c|c|c|c|}
\hline Variables & Coefficient & Standard Error & $P>t$ & $95 \%$ Conf. & Interval \\
\hline \multicolumn{6}{|l|}{$\begin{array}{l}\text { Influential Factors } \\
\text { Causal factors of NCDs }\end{array}$} \\
\hline BMI 30 \& higher (\%) & 3.829 & 2.268 & 0.096 & -0.704 & 8.363 \\
\hline Systolic blood pressure & 4.746 & 7.311 & 0.519 & -9.864 & 19.356 \\
\hline Total cholesterol & 19.273 & 113.257 & 0.865 & -207.053 & 245.600 \\
\hline \multicolumn{6}{|c|}{ Health related infrastructure and economic factors (policy \& program) } \\
\hline Improved sanitation (\%) & -1.806 & 1.076 & 0.098 & -0.395 & 0.345 \\
\hline Poverty (\%) & 0.473 & 0.993 & 0.636 & -1.511 & 2.457 \\
\hline Government healthcare expenditures (\$US) & 791.172 & 377.079 & 0.040 & 37.639 & 1544.704 \\
\hline \multicolumn{6}{|l|}{ Enabling factors (policy \& program) } \\
\hline $\begin{array}{l}\text { General availability of chemotherapy in the public } \\
\text { health system }\end{array}$ & -81.195 & 39.238 & 0.043 & -159.607 & -2.782 \\
\hline Ministry of Health with responsibility for NCDs & -105.139 & 75.371 & 0.168 & -255.756 & 45.479 \\
\hline $\begin{array}{l}\text { General availability of diabetes and electrocardiogram } \\
\text { at primary healthcare level }\end{array}$ & 14.428 & 23.177 & 0.536 & -31.888 & 60.744 \\
\hline \multicolumn{6}{|l|}{ Reinforcing factors (policy \& program) } \\
\hline Implementing NCD management (\$US) & -0.073 & 0.040 & 0.075 & -0.154 & 0.007 \\
\hline $\begin{array}{l}\text { Cardiovascular \& diabetes diseases operational policy/ } \\
\text { strategy/action }\end{array}$ & -113.828 & 61.437 & 0.069 & -236.601 & 8.944 \\
\hline \multicolumn{6}{|l|}{ Predisposing factor (policy \& program) } \\
\hline $\begin{array}{l}\text { Fiscal interventions for knowledge to influence } \\
\text { behaviour change }\end{array}$ & 35.144 & 31.398 & 0.267 & -27.601 & 97.889 \\
\hline constant & 451.504 & 1287.932 & 0.727 & -2111.223 & 3025.232 \\
\hline Number of Observation & 76 & & & & \\
\hline F-statistics $(6,33)$ & 6.33 & & & & \\
\hline Probability >F & 0.000 & & & & \\
\hline R-squared & 0.3391 & & & & \\
\hline Root MSE & 181.22 & & & & \\
\hline
\end{tabular}

Notes: Coefficients variances, and hence standard errors, are estimated by a robust method (Huber/White) that does not assume identically distributed errors. Variance of inflation factors are less than 10 , there is no harmful collinearity among independent variables.

Table 2: Policy influences on NCDs in South Asia and Africa (Regression results).

relatively high systolic blood pressure 130.22 , on an average, among adults aged 25 and above prevails in developing countries. The research finds relatively healthy population aged 25 and above having total cholesterol level 4.4 .

Table 2 shows empirical results of multiple regressions estimated by the method of robust ordinary least squares. The study used the Breusch-Pagan/Cook-Weisberg test for heteroskedasticity and rejected the null hypothesis of homoskedastcity at $\mathrm{chi}^{2}=7.91$ (probability $>\mathrm{chi}^{2}=0.0049$ ). Thus, coefficients variances, and for this reason standard errors, are estimated by a robust method (Huber/White or sandwich estimators) which does not assume identically distributed errors. Variances of inflation factors are less than 10, therefore, there is no harmful collinearity among independent variables.

Concerning the issue of exogenity/endogeneity, the study used the Hausman Specification Test to examine the endogeneity of this empirical model, and examined BMI 30 \& more, systolic blood pressure, and total cholesterol. Under the null hypothesis there is no simultaneity and correlation between $\hat{s}_{i, \text { BMI } 30 \text { \& more }} ; \omega_{\mathrm{i}, \text { systolic blood pressure }}$; and $\hat{\mathrm{s}}_{\mathrm{i}, \text { total cholesterol }}$ and $\mathrm{e}_{\mathrm{i}, \mathrm{NCD}(,)}$ should be zero, asymptotically. The study used four instrument variables: per capita income, alcohol consumption among adults aged 15 and above, health promotion for earmarking taxes from fiscal interventions to influence behavioral changes, and operational strategy or action plan for alcohol use by a government.
Three of the residuals in the structural equation were not found statistically significant at the $10 \%$ level, $\hat{s}_{\mathrm{i}, \mathrm{BMI}} 30$ \& more $(\mathrm{t}=-0.47) ; \omega_{\mathrm{i}, \text { systolic blood }}$

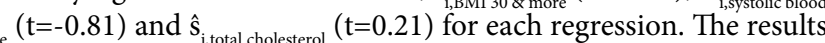
imply that the aforementioned three variables are exogenous variables.

\section{Empirical regression results}

BMI 30 \& higher in the causal factors of NCDs is statistically significant and positively associated with NCDs in the causal factors of Table 2. One percentage increase in BMI 30 \& higher among adults aged 25 and above in the population raises 3.829 NCDs deaths (cardiovascular and diabetes) per 100,000 population (age-standardized estimate among adults) by country. Thus, the increase in NCDs depends on the size of population. Other causal factors -systolic blood pressure and total cholesterol -- are not found statistically significant.

In health related infrastructure and economic factors, the coefficient of government healthcare expenditures require careful interpretation. The variable is actually a reciprocal variable (1/government healthcare expenditures). As the government healthcare expenditures increases indefinitely, the term a(1/government healthcare expenditures) approaches zero and NCDs approaches the limiting mark. The statistically significant value $(=791.172)$ is inversely related to NCDs. A dollar (\$US) increase in government healthcare expenditures per capita per year, reduces NCDs by about 791 persons per 100,000 population per year. Thus, the government costs depend on the population of a 
country. The interpretation, and one of the advantages of the reciprocal approach, is that 791 persons affected by NCDs will reduce to 451.504 persons (constant term in Table 2), which is not statistically significant. Since a government has budget constraints, the target level of optimal NCDs is about 451 NCDs per 100,000 populations per year, and it is not at zero NCDs level. In addition, the reduction cost increases because of diminishing effectiveness of policy. For the result of coefficient about "improved sanitation", the proportion of total population using improved water sources and improved sanitation facilities is statistically significant in the item of health related infrastructure. One percent increase in improved water and sanitation reduces NCDs by 1.806 per 100,000 populations per year. This quantitative effect is moderately small.

For the enabling factor (policy \& program), the health system resources and capabilities are measured as the general availability of chemotherapy in public health systems by country. The estimated impact of chemotherapy in public health systems is that cardiovascular and diabetes deaths per 100,000 populations (age-standardized estimate among adults) by country are 81.195 less than those of non resource/capacity country per year. In the reinforcing factor (policy \& program) in Table 2, implementing key activities related to NCDs management with per capita health expenditure (purchasing power parity current $\$$ US) is statistically significant. An increase of $\$ 1$ reduces 0.073 NCDs incidents per 100,000 populations per year by country and a $10 \%(\$ 25.72)$ increase in NCD management per capita health expenditure reduce(s) $0.22 \%$ cardiovascular and diabetes deaths. Thus, the numerical number is very small but the effectiveness is different from the coefficient.

Another NCD management is whether or not the country has an operational policy/strategy/action plan for cardiovascular diseases and diabetes. The effect is practically large and is statistically significant. The estimated effect of cardiovascular disease/diabetes management is that a country with the operational policy/strategy/action plan for NCDs is 113.828 persons less than those of non-operation country, holding other factors constant. The influence of the specifically targeted cardiovascular disease/diabetes management is relatively larger than other dichotomous factors -- general availability of chemotherapy, which represents access to and availability of healthcare services in the healthcare settings, in the enabling factor (policy \& program).

\section{Discussion}

The study, by using theoretically sound PP model (Figure 1), is the opening of initial quantitative research to shed light on the efficiency of government policies on non-communicable disease (cardiovascular diseases and diabetes) in developing countries. The data for this study is taken from World Heath Statistics: 2008-2010 [5].

The observations comprise 30 developing countries in South Asia and 46 developing countries in Africa. The total number of these observations is 76 , and it is small. Since it makes a small group, the study looks at these observations as a unit of 76 observations. Key findings of this study is that the government public health policies are effective, neither nil or weak quantitatively. The study used three measurement methods which depended on data characteristics: (1) the \$US term, (2) a percentage measure and (3) a dichotomous category. Factors which present statistically significant outcomes are: (1) government healthcare expenditures per capita per year by a country, and an implementing NCD management; (2) BMI 30 \& higher, improved sanitation facilities and improved drinking water sources; and (3) availability of chemotherapy resources in the public health system, and cardiovascular and diabetes diseases operational policy/strategy/action.

\section{Government healthcare expenditures (GHE)}

The interpretation, and one of the advantages of the reciprocal approach, is that 791 persons with the NCDs level decline toward 451.504 persons (constant term in Table 2), which is not statistically significant. Since a government has budget constraints, the target level of optimal NCDs is about 451 NCDs per 100,000 populations per year, and is not zero NCDs. The reduction cost of the government healthcare expenditures will increase because of diminishing effectiveness of policy by decreasing NCD population. Using the results, the study calculates an elasticity of government healthcare expenditures [=coefficient of $\mathrm{GHE}^{\star}$ (reciprocal mean of GHE/mean of NCDs)] and its effect is a $10 \%$ increase in GHE that will lead to a $0.54 \%$ reduction in deaths by NCDs, i.e., cardiovascular diseases and diabetes. A $10 \%$ increase in GHE means $\$ 12.15$ per capita increase per year. Thus the total GHE becomes $\$ 133.60$ [ $=\$ 121.45+\$ 12.15]$. A small increase of the GHE will save about 0.459 million people of developing countries from NCDs. Thus, a well-constructed tax system for financing GHE is required. The study robustly finds the effectiveness of a government's healthcare expenditures. However, we need to understand that the effectiveness is not necessarily to be efficient.

\section{Implementing NCD management (INCDM)}

To assess implementing key activities related to NCD managements with per capita health expenditures (purchasing power parity current \$US), the similar approach is employed. It is the elasticity of NCD managements with per capita health expenditures with respect to deaths from NCDs i.e., cardiovascular diseases and diabetes of NCDs [=variable coefficient of INCDM ${ }^{\star}$ (mean of INCDM/mean of NCDs)] and its effect is a $10 \%$ increase in INCDM will lead to a $0.21 \%$ reduction in deaths from NCDs. The outcome of the policy impact calculated by using the elasticity shows a decrease of 8.921 million NCDs cases in developing countries. The cost of this outcome benefit is an increment of $\$ 25.72$ per person per year. The total cost is $\$ 285.92$ per person per year $[\$ 25.72+\$ 257.20]$. The cost of implementing NCD management is about two times higher than that of the government healthcare expenditures.

\section{Efficiency: Government healthcare expenditures (GHE) vs. Implementing NCD management (INCDM)}

Previous sections reveal the effectiveness of government health policies. This section evaluates the efficiency of aforementioned outcomes. Figure 2 presents concentration curves of the GHE and the INCDM, and the concentration index (CI) of both measures (endnote 1 ). The $\mathrm{CI}$ ranges from -1 to +1 . The value of $\mathrm{CI}$ measures the efficiency of budget allocation/inequality, the higher the absolute number of $\mathrm{CI}$, the grater the inefficiency of budget allocation [16]. The CI equals zero when the concentration curve coincides with the diagonal line that a policy budget allocation is equally distributed in order to reduce NCDs with a constant average cost. If the curve lies above the diagonal line $\mathrm{CI}<0$, and if the curve lies below the diagonal line $\mathrm{CI}>1$. The GHE line suggests that the budget allocation is heavily allocating a large amount of expenditures and deaths from NCDs with given budget allocation. Compared to the GHE line, the INCD line is closer to the equality line than the GHE, the INCDM faces less deaths from NCDs at point "a" than the GHE at a point " $b$ " with given budget allocation. The INCDM is more efficient money allocation than the GHE. The reason is that the policy/program specifically focuses on the reduction of NCDs, i.e. cardiovascular diseases and diabetes. 


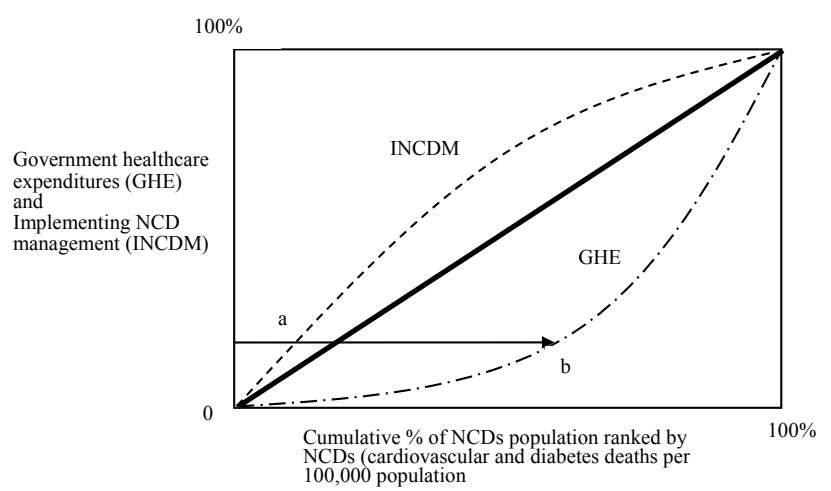

Figure 2: Concentration curves: Non-communicable diseases and government policy programs (government healthcare expenditures vs. implementing NCD management).

Concentration index: Non-communicable diseases

\begin{tabular}{|lrl|c|c|}
\hline Costs & & Concentration index & $\begin{array}{l}\text { S t a n d a rd } \\
\text { errors }\end{array}$ \\
\hline $\begin{array}{l}\text { Government } \\
\text { (GHE) }\end{array}$ & healthcare & expenditures & $0.244 \mathrm{~b}$ & 0.063 \\
\hline $\begin{array}{l}\text { Implementing NCD } \\
\text { (INCDM) }\end{array}$ & management & $-0.062 \mathrm{C}$ & 0.023 \\
\hline
\end{tabular}

Notes: Concentration index uses formula/covariance method.

Standard errors of the concentration index use formula/covariance method.

a, b, and c represent statistically significant levels of $99 \%$ level (a), 95\% level (b), and $90 \%$ level

(c) for a two-tailed test.

\section{BMI 30 \& higher, and improved sanitation facilities and improved drinking water}

The study includes three causal factors of NCDs: BMI 30 and higher, systolic blood pressure and cholesterol ratio [1,17-20]. The study scrutinizes relatively high systolic blood pressure of 130.22 on average, relatively healthy cholesterol level of 4.4 on average, and the average \% of BMI 30 \& higher is $14.32 \%$ by country which is not particularly high. All causal factors are positively associated with the NCDs. However, BMI 30 \& higher is one of the factors that is statistically significant and positively associated with NCDs. In a developing country, urbanization followed by economic development leads to sedentary lifestyle, which increases reliance on public transportation and nutrient-poor diet full of saturated fats. These changes in lifestyle cause obesity, hypertension, and high cholesterol. These are sources of risk/causal factors of cardiovascular diseases and diabetes [17,20-22]. These diseases are almost inevitable under the current urbanization with economic development, especially in developing countries. The effective informal and formal health education regarding NCDs is desirable for health literacy [20]. It is an urgent issue. In addition, from a public health point of view, sanitation and improved water supply systems are necessary with urban planning under the urbanization process [10].

Availability of chemotherapy as the general availability of facility resources in the public health system, and cardiovascular diseases and diabetes operational policy/ strategy/action

Developing countries have non-communicable disease because of their poor environmental characteristics and insufficient medical equipment to deal with emergencies. This study uses general accessibility to chemotherapy facility as an indication of a satisfactory healthcare service. However, developing countries invariably lack this facility. High prevalence of cardiovascular diseases and diabetes stems from unhealthy environment and lifestyle linked with economic development.

Economic development is a double-edged sword. On the one hand it raises income levels of the people, and on the other, creates unhealthy environment through unchecked and unhealthy lifestyles. For cardiovascular diseases and diabetes based on this study, the operational policy/strategy/action is necessary to effectively check against its onslaught on people and to ensure a sustained health improvement plan with particular reference to NCDs. The findings of the results from the cardiovascular and diabetes diseases control by the operational policies/strategies/actions show the strong relationship between effective public policy for NCDs controls and issue-specific strategy for actions.

\section{Conclusion}

The contribution of this study to healthcare literature is that it quantitatively examines the policy and effectiveness of program to check expansion of NCDs. It also carries out efficiency analysis in the framework of a theoretically well founded PP model. The key strength of this study is quantitative outcomes which are useful for decision makers regarding resource allocation for public health programs. Findings of this study highlight the need for paying concerted attention to regulating urban development, population migration matrix, dietary habit, and unhealthy lifestyle associated with increase in income, and need to educate people on health-related subjects. Findings of this study also reveal that the policy/program effectiveness plays a significant role akin to efficiency when resource allocation is scarce. The outcomes of this study pave the way for further examination of NCD policy/ program by using individual data. Suitable survey dates provided by governments in developing countries is essential for assessing growth of a specific illness due to economic progress and concomitant rise in unhealthy lifestyle for policy purposes.

This study is limited in the sense that it focuses only on deaths from NCDs - cardiovascular and diabetes diseases - by using the country level aggregate data. There was a small observation because of limited availability of cross-sectional data and missing values in available observed data, although this study used 76 observation of South Asia and African countries, but findings are significant and helpful in understanding the mechanics of healthcare planning and its repercussions if planning goes wrong and effaces all previous efforts to help improve quality of life a underserved population. The key findings underscore the effectiveness of government NCD policy/program and some good judgment of efficiency by using quantitative regression method and a concentration index approach. As hypothesized, the NCD (cardiovascular and diabetes diseases) policy/program by the governments in developing countries is neither nil or weak. Many developing countries have similar NCDs, but with differing magnitudes. Various NCDs would be prevented by tackling associated and targeted risk factors/health behaviors with informal and formal health policies/ strategies/actions through government initiatives.

\section{Endnote}

The concentration index is computed on individual (micro) data. The concentration index is computed $[23]$ as $\mathrm{CI}=2 \operatorname{cov}\left(\mathrm{h}_{\mathrm{i}}, \mathrm{R}_{\mathrm{i}}\right) / \mu$, where $h_{i}$ is the individual i's indicator variable (e.g. health status) whose inequality is measured, $\mu$ is its mean, and $R_{i}$ is the $i_{\text {th }}$ individual's fractional rank in the socioeconomic distribution (e.g. the person's rank 
Citation: Yamada T, Chen CC, Chiu IM, Rizvi SW (2013) Non-Communicable Diseases in Developing Countries: Causes and Health Policy/Program Assessments. J Trop Dis 1: 117. doi:10.4172/2329-891X.1000117

Page 7 of 7

in the income distribution). $\operatorname{cov}(. .$.$) is the covariance. Standard errors$ of $\mathrm{C}$ are obtained by a covariance/formula method. $\operatorname{var}(\mathrm{CI})=(1 / \mathrm{n})[(1 / \mathrm{n})$ $\left.\sum_{\mathrm{i}=1} \mathrm{a}_{\mathrm{i}}^{2}-(1+\mathrm{CI})^{2}\right]$, where $\mathrm{a}=\left(\mathrm{h}_{\mathrm{i}} / \mu\right)\left(2 \mathrm{R}_{\mathrm{i}}-1-\mathrm{CI}\right)+2-\mathrm{q}_{\mathrm{i}-1}-1-\mathrm{q}_{\mathrm{i}}$, and $\mathrm{q}_{\mathrm{i}}=(1 /$ $\mu \mathrm{n}) \Sigma_{\mathrm{j}=1}\left(\mathrm{~h}_{\mathrm{j}}\right)$ is the ordinate of the concentration curve $\mathrm{CC}_{\mathrm{h}}(\mathrm{p})$, and $\mathrm{q}_{0}=0$.

\section{Acknowledgements}

We are grateful to Joseph Harris of the Rutgers University for the concentration index method. We greatly acknowledge the research support provided by the Research Council of Rutgers University in U.S.A. (\#2-02243 and \#2-02089). Any credits, analyses, interpretations, conclusions and the views expressed in this paper are those of authors, and do not necessarily reflect those of the institutions.

\section{References}

1. Miranda JJ, Kinra S, Casas JP, Davey Smith G, Ebrahim S (2008) Noncommunicable diseases in low- and middle-income countries: context, determinants and health policy. Trop Med Int Health 13: 1225-1234.

2. Boutayeb A, Boutayeb S (2005) The burden of non communicable diseases in developing countries. Int J Equity Health 4: 2.

3. Mayosi BM, Flisher AJ, Lalloo UG, Sitas F, Tollman SM, et al. (2009) The burden of non-communicable diseases in South Africa. Lancet 374: 934-947.

4. World Economic Forum (2011) Global economic burden of non-communicable diseases. WHO.

5. World Health Statistics (2012) WHO, Geneva, Switzerland.

6. Hossain P, Kawar B, EI Nahas M (2007) Obesity and diabetes in the developing world--a growing challenge. N Engl J Med 356: 213-215.

7. Abubakari AR, Lauder W, Jones MC, Kirk A, Agyemang C, et al. (2009) Prevalence and time trends in diabetes and physical inactivity among adult West African populations: the epidemic has arrived. Public Health 123: 602614.

8. Wagner $\mathrm{KH}$, Brath $\mathrm{H}$ (2012) A global view on the development on non communicable diseases. Prev Med 54: S38-S41.

9. Dumith SC, Muniz LC, Tassitano RM, Hallal PC, Menezes AM (2012) Clustering of risk factors for chronic diseases among adolescents from Southern Brazil. Prev Med 54: 393-396.

10. Vaidya A, Shakya S, Krettek A (2010) Obesity prevalence in Nepal: public health challenges in a low-income nation during an alarming worldwide trend. Int J Environ Res Public Health 7: 2726-2744.
11. Tagoe HA, Dake FA (2011) Healthy lifestyle behaviour among Ghanaian adults in the phase of a health policy change. Global Health $7: 7$.

12. Green, LW, Kreuter, MW (2005) Health Program Planning: An Educational and Ecological Approach (4th ed.). New York, USA: McGraw-Hill.

13. Yamada T, Chen CC, Yamada T (2005) Economic evaluation of relapse prevention for substance users: treatment settings and health care policy. Adv Health Econ Health Serv Res 16: 431-450.

14. Gujarati D (2011) Econometrics. New York, USA: Palgrave Macmillan.

15. Koolman X, van Doorslaer E (2004) On the interpretation of a concentration index of inequality. Health Econ 13: 649-656.

16. Zhang Q, Wang $Y$ (2007) Using concentration index to study changes in socioeconomic inequality of overweight among US adolescents between 1971 and 2002. Int J Epidemiol 36: 916-925.

17. Ezeamama AE, Viali S, Tuitele J, McGarvey ST (2006) The influence of socioeconomic factors on cardiovascular disease risk factors in the context of economic development in the Samoan archipelago. Soc Sci Med 63: 25332545.

18. Ismail MN, Chee SS, Nawawi H, Yusoff, K, Lim TO et al. (2002) Obesity in Malaysia.

19. Laux TS, Bert PJ, González M, Unruh M, Aragon A, et al. (2012) Prevalence of obesity, tobacco use, and alcohol consumption by socioeconomic status among six communities in Nicaragua. Rev Panam Salud Publica 32: 217-225.

20. Rajadurai J, Lopez EA, Rahajoe AU, Goh PP, Uboldejpracharak Y, et al. (2012) Women's cardiovascular health: perspectives from South-East Asia. Nat Rev Cardiol 9: 464-477.

21. Allender S, Lacey B, Webster P, Rayner M, Deepa M, et al. (2010) Level of urbanization and noncommunicable disease risk factors in Tamil Nadu, India. Bull World Health Organ 88: 297-304.

22. Allender S, Wickramasinghe K, Goldacre M, Matthews D, Katulanda P (2011) Quantifying urbanization as a risk factor for noncommunicable disease. J Urban Health 88: 906-918.

23. Kakwani NC, Wagstaff A, van Doorslaer E (1997) Socioeconomic inequalities in health: measurement, computation, and statistical inference. Journal of Econometrics 77: 87-103. 